\title{
LOWER SEMICONTINUOUS FUNCTIONALS FOR ALMGREN'S MULTIPLE VALUED FUNCTIONS
}

\section{Camillo De Lellis, Matteo Focardi and Emanuele Nunzio Spadaro}

\author{
Universität Zürich, Institut für Mathematik \\ Winterthurerstrasse 190, CH-8057 Zürich, Schweiz; camillo.delellis@math.uzh.ch \\ Università di Firenze, Dipartimento di Matematica "Ulisse Dini" \\ Viale Morgagni, 67/a, 50134 Firenze, Italia; focardi@math.unifi.it \\ Universität Bonn, Hausdorff Center for Mathematics
}

Villa Maria, Endenicher Allee 62, D-53115 Bonn, Germany; emanuele.spadaro@hcm.uni-bonn.de

\begin{abstract}
We consider general integral functionals on the Sobolev spaces of multiple valued functions introduced by Almgren. We characterize the semicontinuous ones and recover earlier results of Mattila in [10] as a particular case. Moreover, we answer positively to one of the questions raised by Mattila in the same paper.
\end{abstract}

\section{Introduction}

In his big regularity paper [1], Almgren developed a new theory of weakly differentiable multiple valued maps minimizing a suitable generalization of the classical Dirichlet energy. He considered maps defined on a Lipschitz domain $\Omega \subset \mathbf{R}^{m}$ and taking values in the space of $Q$ unordered points of $\mathbf{R}^{n}$, which minimize the integral of the squared norm of the derivative (conveniently defined). The regularity theory for these so called Dir-minimizing $Q$-valued maps is a cornerstone in his celebrated proof that the Hausdorff dimension of the singular set of an $m$-dimensional area-minimizing current is at most $(m-2)$.

The existence of Dir-minimizing functions with prescribed boundary data is proven in [1] via the direct method in the calculus of variations. Thus, the generalized Dirichlet energy is semicontinuous under weak convergence. This property is not specific of the energy considered by Almgren. Mattila in [10] considered some energies induced by homogeneous quadratic polynomials of the partial derivatives. His energies are the first non-constant term in the Taylor expansion of elliptic geometric integrands and hence generalize Almgren's Dirichlet functional, which is the first non-constant term in the expansion of the area functional.

Mattila showed that these quadratic functionals are lower semicontinuous under weak convergence. A novelty in Mattila's work was the impossibility to use Almgren's extrinsic biLipschitz embeddings of the space of $Q$-points into a Euclidean space, because of the more complicated form of the energies (cp. with [1] and [5] for the existence and properties of these embeddings). In this paper we push forward the investigation of Mattila and, taking advantage of the intrinsic metric theory for $Q$ valued functions developed in [5], we generalize his results to the case of general integral functionals defined on Sobolev spaces of $Q$-functions. We obtain a complete

doi:10.5186/aasfm.2011.3626

2010 Mathematics Subject Classification: Primary 49J45, 49Q20.

Key words: $Q$-valued functions, semicontinuity, quasiconvexity, $Q$-ellipticity. 
characterization of the semicontinuity and a simple criterion to recognize efficiently a specific class of semicontinuous functionals. Mattila's $Q$-semielliptic energies fall obviously into this class. Indeed, a simple corollary of our analysis is that a quadratic energy as considered in [10] is $Q$-semielliptic if and only if it is quasiconvex (see Definition 0.1 and Remark 2.1 for the relevant definitions). Moreover, in the special cases of dimensions $m=2$ or $n=2$, we can answer positively to the question posed by Mattila himself on the equivalence of $Q$-semiellipticity and 1-semiellipticity.

0.1. Quasiconvexity and lower semicontinuity. In order to illustrate the results, we introduce the following terminology (we refer to [5] and Subsection 1.1 for the relevant definitions and terminology concerning $Q$-valued maps).

Let $\Omega \subset \mathbf{R}^{m}$ be a bounded open set. A measurable map $f: \Omega \times\left(\mathbf{R}^{n}\right)^{Q} \times$ $\left(\mathbf{R}^{m \times n}\right)^{Q} \rightarrow \mathbf{R}$ is called a $Q$-integrand if, for every permutation $\pi$ of $\{1, \ldots, Q\}$,

$$
f\left(x, a_{1}, \ldots, a_{Q}, A_{1}, \ldots, A_{Q}\right)=f\left(x, a_{\pi(1)}, \ldots, a_{\pi(Q)}, A_{\pi(1)}, \ldots, A_{\pi(Q)}\right) .
$$

Note that, by (1.2) (see also [5, Remark 1.11]), given a weakly differentiable $Q$-valued map $u$, the expression $f(\cdot, u, D u)=f\left(\cdot, u_{1}, \ldots, u_{Q}, D u_{1}, \ldots, D u_{Q}\right)$ is well defined almost everywhere in $\Omega$. Thus, for any Sobolev $Q$-valued function the following energy makes sense:

$$
F(u)=\int_{\Omega} f(x, u(x), D u(x)) d x .
$$

Our characterization of (weakly) lower-semicontinuous functionals $F$ is the counterpart of Morrey's celebrated result in the vectorial calculus of the variations (see [11], [12]). We start by introducing the relevant notion of quasiconvexity, which is a suitable generalization of Morrey's definition. From now on we set $C_{r}:=$ $[-r / 2, r / 2]^{m}$.

Definition 0.1. (Quasiconvexity) Let $f:\left(\mathbf{R}^{n}\right)^{Q} \times\left(\mathbf{R}^{m \times n}\right)^{Q} \rightarrow \mathbf{R}$ be a locally bounded $Q$-integrand. We say that $f$ is quasiconvex if the following holds for every affine $Q$-valued function $u(x)=\sum_{j=1}^{J} q_{j} \llbracket a_{j}+L_{j} \cdot x \rrbracket$, with $a_{i} \neq a_{j}$ for $i \neq j$. Given any collection of maps $w^{j} \in W^{1, \infty}\left(C_{1}, \mathscr{A}_{q_{j}}\right)$ with $\left.w^{j}\right|_{\partial C_{1}}=q_{j} \llbracket a_{j}+\left.L_{j}\right|_{\partial C_{1}} \rrbracket$ we have the inequality

$$
f(u(0), D u(0)) \leq \int_{C_{1}} f(\underbrace{a_{1}, \ldots, a_{1}}_{q_{1}}, \ldots, \underbrace{a_{J}, \ldots, a_{J}}_{q_{J}}, D w^{1}(x), \ldots, D w^{J}(x)) d x .
$$

The main result is the following.

Theorem 0.2. Let $p \in\left[1, \infty\left[\right.\right.$ and $f: \Omega \times\left(\mathbf{R}^{n}\right)^{Q} \times\left(\mathbf{R}^{m \times n}\right)^{Q} \rightarrow \mathbf{R}$ be a continuous $Q$-integrand. If $f(x, \cdot, \cdot)$ is quasiconvex for every $x \in \Omega$ and

$$
0 \leq f(x, a, A) \leq C\left(1+|a|^{q}+|A|^{p}\right) \quad \text { for some constant } C,
$$

where $q=0$ if $p>m, q=p^{*}$ if $p<m$ and $q \geq 1$ is any exponent if $p=m$, then the functional $F$ in $(0.1)$ is weakly lower semicontinuous in $W^{1, p}\left(\Omega, \mathscr{A}_{Q}\left(\mathbf{R}^{n}\right)\right)$. Conversely, if $F$ is weakly* lower semicontinuous in $W^{1, \infty}\left(\Omega, \mathscr{A}_{Q}\left(\mathbf{R}^{n}\right)\right)$, then $f(x, \cdot, \cdot)$ is quasiconvex for every $x \in \Omega$.

Remark 0.3. It is easy to see that a quadratic integrand is $Q$-semielliptic in the sense of Mattila if and only if it is quasiconvex, cp. to Remark 2.1. 
0.2. Polyconvexity. We continue to follow the classical path of the vectorial calculus of variations and introduce a suitable generalization of the well-known notion of polyconvexity (see [12], [2]). Let $N:=\min \{m, n\}, \tau(n, m):=\sum_{k=1}^{N}\left(\begin{array}{l}m \\ k\end{array}\right)\left(\begin{array}{l}n \\ k\end{array}\right)$ and define $M: \mathbf{R}^{n \times m} \rightarrow \mathbf{R}^{\tau(m, n)}$ as $M(A):=\left(A, \operatorname{adj}_{2} A, \ldots, \operatorname{adj}_{N} A\right)$, where $\operatorname{adj}_{k} A$ stands for the matrix of all $k \times k$ minors of $A$.

Definition 0.4. A $Q$-integrand $f:\left(\mathbf{R}^{n}\right)^{Q} \times\left(\mathbf{R}^{n \times m}\right)^{Q} \rightarrow \mathbf{R}$ is polyconvex if there exists a map $g:\left(\mathbf{R}^{n}\right)^{Q} \times\left(\mathbf{R}^{\tau(m, n)}\right)^{Q} \rightarrow \mathbf{R}$ such that:

(i) the function $g\left(a_{1}, \ldots, a_{Q}, \cdot\right):\left(\mathbf{R}^{\tau(m, n)}\right)^{Q} \rightarrow \mathbf{R}$ is convex for every $a_{1}, \ldots, a_{Q} \in$ $\mathbf{R}^{n}$

(ii) for every $a_{1}, \ldots, a_{Q} \in \mathbf{R}^{n}$ and $\left(L_{1}, \ldots, L_{Q}\right) \in\left(\mathbf{R}^{n \times m}\right)^{Q}$ it holds

$$
f\left(a_{1}, \ldots, a_{Q}, L_{1}, \ldots, L_{Q}\right)=g\left(a_{1}, \ldots, a_{Q}, M\left(L_{1}\right), \ldots, M\left(L_{Q}\right)\right) .
$$

Polyconvexity is much easier to verify. For instance, if $\min \{m, n\} \leq 2$, quadratic integrands are polyconvex if and only if they are 1-semielliptic in the sense of Mattila, cp. to Remark 3.4. Combining this with Remark 0.3 and Theorem 0.5, we easily conclude that $Q$-semiellipticity and 1-semiellipticity coincide in this case, as suggested by Mattila himself in [10].

Theorem 0.5. Every locally bounded polyconvex $Q$-integrand $f$ is $Q$-quasiconvex.

For integrands on single valued maps, the classical proof of Theorem 0.5 relies on suitable integration by parts formulas, called Piola's identities by some authors. These identities can be shown by direct computation. However, an elegant way to derive them is to rewrite the quantities involved as integrals of suitable differential forms over the graph of the given map. The integration by parts is then explained via Stokes' Theorem. This point of view is the starting of the theory of Cartesian currents developed by Giaquinta, Modica and Souček (see the monograph [8, 9]). Here we take this approach to derive similar identities in the case of $Q$-valued maps, building on the obvious structure of current induced by the graph of Lipschitz $Q$ valued maps $f: \Omega \rightarrow \mathscr{A}_{Q}\left(\mathbf{R}^{n}\right)$ (which we denote by gr $(f)$ ). A key role is played by the intuitive identity $\partial \operatorname{gr}(f)=\operatorname{gr}\left(\left.f\right|_{\partial \Omega}\right)$, which for $Q$-valued maps is less obvious. A rather lengthy proof of this fact was given for the first time in [1]. We refer to Appendix C of [4] for a much shorter derivation. A final comment is in order. Due to the combinatorial complexity of $Q$-valued maps, we do not know whether Theorem 0.5 can be proved without using the theory of currents.

The paper is organized in three sections. The first one contains three technical lemmas on $Q$-valued Sobolev functions, proved using the language of [5] (which differs slightly from Almgren's original one). In Section 2 we prove Theorem 0.2 and in Section 3 Theorem 0.5. In the appendix we collect some results on equi-integrable functions, essentially small variants of Chacon's biting lemma, which have already appeared in the literature: we include their proofs for reader's convenience.

\section{Q-valued functions}

In this section we recall the notation and terminology of [5], and provide some preliminary results which will be used in the proofs of Theorem 0.2 and Theorem 0.5. 
1.1. Sobolev $Q$-valued functions. $Q$-valued functions are maps valued in the complete metric space of unordered sets of $Q$ points in $\mathbf{R}^{n}$.

Definition 1.1. We denote by $\left(\mathscr{A}_{Q}\left(\mathbf{R}^{n}\right), \mathscr{G}\right)$ the metric space of unordered $Q$ tuples given by

$$
\mathscr{A}_{Q}\left(\mathbf{R}^{n}\right):=\left\{\sum_{i=1}^{Q} \llbracket P_{i} \rrbracket: P_{i} \in \mathbf{R}^{n} \text { for every } i=1, \ldots, Q\right\},
$$

where $\llbracket P_{i} \rrbracket$ denotes the Dirac mass in $P_{i} \in \mathbf{R}^{n}$ and

$$
\mathscr{G}\left(T_{1}, T_{2}\right):=\min _{\sigma \in \mathscr{P}_{Q}} \sqrt{\sum_{i}\left|P_{i}-S_{\sigma(i)}\right|^{2}}
$$

with $T_{1}=\sum_{i} \llbracket P_{i} \rrbracket$ and $T_{2}=\sum_{i} \llbracket S_{i} \rrbracket \in \mathscr{A}_{Q}\left(\mathbf{R}^{n}\right)$, and $\mathscr{P}_{Q}$ denotes the group of permutations of $\{1, \ldots, Q\}$.

Given a vector $v \in \mathbf{R}^{n}$, we denote by $\tau_{v}(T)$ the translation of the $Q$-point $T=$ $\sum_{i} \llbracket T_{i} \rrbracket$ under $v$ given by

$$
\tau_{v}(T):=\sum_{i} \llbracket T_{i}-v \rrbracket .
$$

Continuous, Lipschitz, Hölder and (Lebesgue) measurable functions from $\Omega$ into $\mathscr{A}_{Q}$ are defined in the usual way. It is a general fact that any measurable $Q$-valued function $u: \Omega \rightarrow \mathscr{A}_{Q}$ can be written as the "sum" of $Q$-measurable functions $u_{1}, \ldots, u_{Q}$ [5, Proposition 0.4]:

$$
u(x)=\sum_{i} \llbracket u_{i}(x) \rrbracket \quad \text { for a.e. } x \in \Omega .
$$

We now recall the definition of the Sobolev spaces of functions taking values in the metric space of $Q$-points.

Definition 1.2. A measurable $u: \Omega \rightarrow \mathscr{A}_{Q}$ is in the Sobolev class $W^{1, p}, 1 \leq p \leq$ $\infty$, if there exists $\varphi \in L^{p}(\Omega ;[0,+\infty))$ such that

(i) $x \mapsto \mathscr{G}(u(x), T) \in W^{1, p}(\Omega)$ for all $T \in \mathscr{A}_{Q}$;

(ii) $|D \mathscr{G}(u, T)| \leq \varphi$ a.e. in $\Omega$ for all $T \in \mathscr{A}_{Q}$.

As for classical Sobolev maps, an important feature of Sobolev $Q$-valued functions is the existence of the approximate differential almost everywhere. Given $u \in W^{1, p}\left(\Omega, \mathscr{A}_{Q}\left(\mathbf{R}^{n}\right)\right)$, there exists a $Q$ map $D u=\sum_{i} \llbracket D u_{i} \rrbracket: \Omega \rightarrow \mathscr{A}_{Q}\left(\mathbf{R}^{m \times n}\right)$ such that, for almost every $x_{0} \in \Omega$, the first order approximation

$$
T_{x_{0}} u(x):=\sum_{i} \llbracket D u_{i}\left(x_{0}\right) \cdot\left(x-x_{0}\right)+u_{i}\left(x_{0}\right) \rrbracket
$$

satisfies the following:

(i) there exists a set $\tilde{\Omega}$ with density one at $x_{0}$ such that $\mathscr{G}\left(u(x), T_{x_{0}} u\right)=o\left(\left|x-x_{0}\right|\right)$ as $x \rightarrow x_{0}, x \in \tilde{\Omega}$

(ii) $D u_{i}\left(x_{0}\right)=D u_{j}\left(x_{0}\right)$ if $u_{i}\left(x_{0}\right)=u_{j}\left(x_{0}\right)$.

Moreover, the map $D u$ is $L^{p}$ integrable, meaning that

$$
|D u|:=\sqrt{\sum_{i}\left|D u_{i}\right|^{2}} \in L^{p}(\Omega) .
$$


Finally, we recall the definition of weak convergence in $W^{1, p}\left(\Omega, \mathscr{A}_{Q}\left(\mathbf{R}^{n}\right)\right)$.

Definition 1.3. Let $u_{k}, u \in W^{1, p}\left(\Omega ; \mathscr{A}_{Q}\right)$. We say that $u_{k}$ converges weakly to $u$ for $k \rightarrow \infty$, (and we write $u_{k} \rightarrow u$ ) in $W^{1, p}\left(\Omega ; \mathscr{A}_{Q}\right)$, if

(i) $\int \mathscr{G}\left(f_{k}, f\right)^{p} \rightarrow 0$, for $k \rightarrow \infty$;

(ii) $\sup _{k} \int\left|D f_{k}\right|^{p}<\infty$.

1.2. $L^{p}$-approximate differentiability. Here we prove a more refined differentiability result.

Lemma 1.4. Let $u \in W^{1, p}\left(\Omega, \mathscr{A}_{Q}\right)$. Then, for $\mathscr{L}^{m}$-a.e. $x_{0} \in \Omega$ it holds

$$
\lim _{\rho \rightarrow 0} \rho^{-p-m} \int_{C_{\rho}\left(x_{0}\right)} \mathscr{G}^{p}\left(u, T_{x_{0}} u\right)=0 .
$$

Proof. By the Lipschitz approximation in [5, Proposition 4.4], there exists a family of functions $\left(u_{\lambda}\right)$ such that:

(a) $\operatorname{Lip}\left(u_{\lambda}\right) \leq \lambda$ and $d_{W^{1, p}}\left(u, u_{\lambda}\right)=o(1)$ as $\lambda \rightarrow+\infty$;

(b) the sets $\Omega_{\lambda}=\left\{x: T_{x} u=T_{x} u_{\lambda}\right\}$ satisfy $\Omega_{\lambda} \subset \Omega_{\lambda^{\prime}}$ for $\lambda<\lambda^{\prime}$ and $\mathscr{L}^{m}\left(\Omega \backslash \Omega_{\lambda}\right)=$ $o(1)$ as $\lambda \rightarrow+\infty$.

We prove (1.3) for the points $x_{0} \in \Omega_{\lambda}$ which are Lebesgue points for $\chi_{\Omega_{\lambda}}$ and $|D u|^{p} \chi_{\Omega \backslash \Omega_{\lambda}}$, for some $\lambda \in \mathbf{N}$, that is

$$
\lim _{\rho \rightarrow 0} f_{C_{\rho}\left(x_{0}\right)} \chi_{\Omega_{\lambda}}=1 \text { and } \lim _{\rho \rightarrow 0} f_{C_{\rho}\left(x_{0}\right)}|D u|^{p} \chi_{\Omega \backslash \Omega_{\lambda}}=0 .
$$

Let, indeed, $x_{0}$ be a point as in (1.4) for a fixed $\Omega_{\lambda}$. Then,

$$
\begin{aligned}
f_{C_{\rho}\left(x_{0}\right)} \mathscr{G}^{p}\left(u, T_{x_{0}} u\right) & \leq 2^{p-1} f_{C_{\rho}\left(x_{0}\right)} \mathscr{G}^{p}\left(u_{\lambda}, T_{x_{0}} u_{\lambda}\right)+2^{p-1} f_{C_{\rho}\left(x_{0}\right)} \mathscr{G}^{p}\left(u_{\lambda}, u\right) \\
& \leq o\left(\rho^{p}\right)+C \rho^{p-m} \int_{C_{\rho}\left(x_{0}\right) \backslash \Omega_{\lambda}}\left|D\left(\mathscr{G}\left(u_{\lambda}, u\right)\right)\right|^{p},
\end{aligned}
$$

where in the latter inequality we used Rademacher's theorem for $Q$-functions (see [5, Theorem 1.13]) and a Poincaré inequality for the classical Sobolev function $\mathscr{G}\left(u, u_{\lambda}\right)$ which by (1.4) satisfies

$$
\Omega_{\lambda} \subseteq\left\{\mathscr{G}\left(u, u_{\lambda}\right)=0\right\} \quad \text { and } \quad \rho^{-m} \mathscr{L}^{m}\left(C_{\rho}\left(x_{0}\right) \cap \Omega_{\lambda}\right) \geq 1 / 2 \text { for small } \rho .
$$

Since $\mathscr{G}\left(u, u_{\lambda}\right)=\sup _{T_{i}}\left|\mathscr{G}\left(u, T_{i}\right)-\mathscr{G}\left(T_{i}, u_{\lambda}\right)\right|$ and

$$
D\left|\mathscr{G}\left(u, T_{i}\right)-\mathscr{G}\left(T_{i}, u_{\lambda}\right)\right| \leq\left|D \mathscr{G}\left(u, T_{i}\right)\right|+\left|D \mathscr{G}\left(T_{i}, u_{\lambda}\right)\right| \leq|D u|+\left|D u_{\lambda}\right| \quad \mathscr{L}^{m} \text {-a.e. on } \Omega \text {, }
$$

we conclude (recall that $\lambda \leq C|D u|$ on $\Omega \backslash \Omega_{\lambda}$ )

$$
\begin{aligned}
\rho^{p-m} \int_{C_{\rho}\left(x_{0}\right) \backslash \Omega_{\lambda}}\left|D\left(\mathscr{G}\left(u, u_{\lambda}\right)\right)\right|^{p} & \leq \rho^{p-m} \int_{C_{\rho}\left(x_{0}\right) \backslash \Omega_{\lambda}} \sup _{i}\left(D\left|\mathscr{G}\left(u, T_{i}\right)-\mathscr{G}\left(T_{i}, u_{\lambda}\right)\right|\right)^{p} \\
& \leq C \rho^{p-m} \int_{C_{\rho}\left(x_{0}\right) \backslash \Omega_{\lambda}}|D u|^{p} \stackrel{(1.4)}{=} o\left(\rho^{p}\right),
\end{aligned}
$$

which finishes the proof.

1.3. Equi-integrability. In the first lemma we show how a weakly convergent sequence of $Q$-functions can be truncated in order to obtain an equi-integrable sequence still weakly converging to the same limit. This result is the analog of $[7$, 
Lemma 2.3] for $Q$-valued functions and constitute a main point in the proof of the sufficiency of quasiconvexity for the lower semicontinuity. Details on equi-integrability can be found in the Appendix.

Lemma 1.5. Let $\left(v_{k}\right) \subset W^{1, p}\left(\Omega, \mathscr{A}_{Q}\right)$ be weakly converging to $u$. Then, there exists a subsequence $\left(v_{k_{j}}\right)$ and a sequence $\left(u_{j}\right) \subset W^{1, \infty}\left(\Omega, \mathscr{A}_{Q}\right)$ such that

(i) $\mathscr{L}^{m}\left(\left\{v_{k_{j}} \neq u_{j}\right\}\right)=o(1)$ and $u_{j} \rightarrow u$ in $W^{1, p}\left(\Omega, \mathscr{A}_{Q}\right)$;

(ii) $\left(\left|D u_{j}\right|^{p}\right)$ is equi-integrable;

(iii) if $p \in[1, m),\left(\left|u_{j}\right|^{p^{*}}\right)$ is equi-integrable and, if $p=m,\left(\left|u_{j}\right|^{q}\right)$ is equi-integrable for any $q \geq 1$.

Proof. Let $g_{k}:=M^{p}\left(\left|D v_{k}\right|\right)$ and notice that, by the estimate on the maximal function operator (see [13] for instance), $\left(g_{k}\right) \subset L^{1}(\Omega)$ is a bounded sequence. Applying Chacon's biting lemma (see Lemma A.2 in the Appendix) to $\left(g_{k}\right)$, we get a subsequence $\left(k_{j}\right)$ and a sequence $t_{j} \nearrow+\infty$ such that $\left(g_{k_{j}} \wedge t_{j}\right)$ are equi-integrable.

Let $\Omega_{j}:=\left\{x \in \Omega: g_{k_{j}}(x) \leq t_{j}\right\}$ and $u_{j}$ be the Lipschitz extension of $\left.v_{k_{j}}\right|_{\Omega_{j}}$ with Lipschitz constant $c t_{j}^{1 / p}$ (see [5, Theorem 1.7]). Then, following [5, Proposition 4.4], it is easy to verify that $\mathscr{L}^{m}\left(\Omega \backslash \Omega_{j}\right)=o\left(t_{j}^{-1}\right)$ and $d_{W^{1, p}}\left(u_{j}, v_{k_{j}}\right)=o(1)$. Thus, (i) follows immediately from these properties and (ii) from

$$
\left|D u_{j}\right|^{p}=\left|D v_{k_{j}}\right|^{p} \leq g_{k_{j}} \wedge t_{j} \text { on } \Omega_{j} \quad \text { and } \quad\left|D u_{j}\right|^{p} \leq c t_{j}=c\left(g_{k_{j}} \wedge t_{j}\right) \text { on } \Omega \backslash \Omega_{j} .
$$

As for (iii), note that the functions $f_{j}:=\mathscr{G}\left(u_{j}, Q \llbracket 0 \rrbracket\right)$ are in $W^{1, p}(\Omega)$, with $\left|D f_{j}\right| \leq$ $\left|D u_{j}\right|$ by the very definition of metric space valued Sobolev maps. Moreover, by (i), $f_{j}$ converge weakly to $|u|$, since $\left\||u|-f_{j}\right\|_{L^{p}} \leq\left\|\mathscr{G}\left(u, u_{j}\right)\right\|_{L^{p}}$. Hence, $\left(\left|f_{j}\right|^{p}\right)$ and $\left(\left|D f_{j}\right|^{p}\right)$ are equi-integrable. In case $p \in[1, m)$, this implies (see Lemma A.3) the equi-integrability of $\left(\left|u_{j}\right|^{p^{*}}\right)$. In case $p=m$, the property follows from Hölder inequality and Sobolev embedding (details are left to the reader).

1.4. Averaged equi-integrability. The next lemma gives some properties of sequences of functions whose blow-ups are equi-integrable. In what follows a function $\varphi:[0,+\infty] \rightarrow[0,+\infty]$ is said superlinear at infinity if $\lim _{t \uparrow+\infty} \frac{\varphi(t)}{t}=+\infty$.

Lemma 1.6. Let $g_{k} \in L^{1}(\Omega)$ with $g_{k} \geq 0$ and $\sup _{k} f_{C_{\rho_{k}}} \varphi\left(g_{k}\right)<+\infty$, where $\rho_{k} \downarrow 0$ and $\varphi$ is superlinear at infinity. Then, it holds

$$
\lim _{t \rightarrow+\infty}\left(\sup _{k} \rho_{k}^{-m} \int_{\left\{g_{k} \geq t\right\}} g_{k}\right)=0
$$

and, for sets $A_{k} \subseteq C_{\rho_{k}}$ such that $\mathscr{L}^{m}\left(A_{k}\right)=o\left(\rho_{k}^{m}\right)$,

$$
\lim _{k \rightarrow+\infty} \rho_{k}^{-m} \int_{A_{k}} g_{k}=0
$$

Proof. Using the superlinearity of $\varphi$, for every $\varepsilon>0$ there exists $R>0$ such that $t \leq \varepsilon \varphi(t)$ for every $t \geq R$, so that

$$
\limsup _{t \rightarrow+\infty}\left(\sup _{k} \rho_{k}^{-m} \int_{\left\{g_{k} \geq t\right\}} g_{k}\right) \leq \varepsilon \sup _{k} f_{C_{\rho_{k}}} \varphi\left(g_{k}\right) \leq C \varepsilon .
$$

Then, (1.6) follows as $\varepsilon \downarrow 0$. For what concerns (1.7), we have

$$
\rho_{k}^{-m} \int_{A_{k}} g_{k}=\rho_{k}^{-m} \int_{A_{k} \cap\left\{g_{k} \leq t\right\}} g_{k}+\rho_{k}^{-m} \int_{A_{k} \cap\left\{g_{k} \geq t\right\}} g_{k} \leq t \rho_{k}^{-m} \mathscr{L}^{m}\left(A_{k}\right)+\sup _{k} \rho_{k}^{-m} \int_{\left\{g_{k} \geq t\right\}} g_{k} \text {. }
$$


By the hypothesis $\mathscr{L}^{m}\left(A_{k}\right)=o\left(\rho_{k}^{m}\right)$, taking the limit as $k$ tends to $+\infty$ and then as $t$ tends to $+\infty$, by (1.6) the right hand side above vanishes.

1.5. Push-forward of currents under $Q$-functions. We define now the integer rectifiable current associated to the graph of a $Q$-valued function. As for Lipschitz single valued functions, we can associate to the graph of a Lipschitz $Q-$ function $u: \Omega \rightarrow \mathscr{A}_{Q}$ a rectifiable current $T_{u, \Omega}$ defined by

$$
\left\langle T_{u, \Omega}, \omega\right\rangle=\int_{\Omega} \sum_{i}\left\langle\omega\left(x, u_{i}(x)\right), \vec{T}_{u_{i}}(x)\right\rangle d \mathscr{H}^{m}(x) \quad \forall \omega \in \mathscr{D}^{m}\left(\mathbf{R}^{m+n}\right),
$$

where $\vec{T}_{u_{i}}(x)$ is the $m$-vector given by $\left(e_{1}+\partial_{1} u_{i}(x)\right) \wedge \cdots \wedge\left(e_{m}+\partial_{m} u_{i}(x)\right) \in \Lambda_{m}\left(\mathbf{R}^{m+n}\right)$. In coordinates, writing $\omega(x, y)=\sum_{l=1}^{N} \sum_{|\alpha|=|\beta|=l} \omega_{\alpha \beta}^{l}(x, y) d x_{\bar{\alpha}} \wedge d y_{\beta}$, where $\bar{\alpha}$ denotes the complementary multi-index of $\alpha$, the current $T_{u, \Omega}$ acts in the following way:

$$
\left\langle T_{u, \Omega}, \omega\right\rangle=\int_{\Omega} \sum_{i=1}^{Q} \sum_{l=1}^{N} \sum_{|\alpha|=|\beta|=l} \sigma_{\alpha} \omega_{\alpha \beta}^{l}\left(x, u_{i}(x)\right) M_{\alpha \beta}\left(D u_{i}(x)\right) d x,
$$

with $\sigma_{\alpha} \in\{-1,1\}$ the sign of the permutation ordering $(\alpha, \bar{\alpha})$ in the natural increasing order and $M_{\alpha \beta}(A)$ denoting the $\alpha, \beta$ minor of a matrix $A \in \mathbf{R}^{n \times m}$,

$$
M_{\alpha \beta}(A):=\operatorname{det}\left(\begin{array}{ccc}
A_{\alpha_{1} \beta_{1}} & \ldots & A_{\alpha_{1} \beta_{k}} \\
\vdots & \ddots & \vdots \\
A_{\alpha_{k} \beta_{1}} & \ldots & A_{\alpha_{k} \beta_{k}}
\end{array}\right)
$$

Analogously, assuming that $\Omega$ is a Lipschitz domain, using parametrizations of the boundary, one can define the current associate to the graph of $u$ restricted to $\partial \Omega$, and both $T_{u, \Omega}$ and $T_{u, \partial \Omega}$ turn out to be rectifiable current-see [4, Appendix C]. The main result about the graphs of Lipschitz $Q$-functions we are going to use is the following theorem proven in [4, Theorem C.3].

Theorem 1.7. For every $\Omega$ Lipschitz domain and $u \in \operatorname{Lip}\left(\Omega, \mathscr{A}_{Q}\right), \partial T_{u, \Omega}=$ $T_{u, \partial \Omega}$.

\section{Quasiconvexity and lower semicontinuity}

In this section we prove Theorem 0.2. Before starting, we link our notion of quasiconvexity with the $Q$-semiellipticity introduced in [10].

Remark 2.1. Following Mattila, a quadratic integrand is a function of the form

$$
E(u):=\int_{\Omega} \sum_{i}\left\langle A D u_{i}, D u_{i}\right\rangle,
$$

where $\mathbf{R}^{n \times m} \ni M \mapsto A M \in \mathbf{R}^{n \times m}$ is a linear symmetric map. This integrand is called $Q$-semielliptic if

$$
\int_{\mathbf{R}^{m}} \sum_{i}\left\langle A D f_{i}, D f_{i}\right\rangle \geq 0 \quad \forall f \in \operatorname{Lip}\left(\mathbf{R}^{m}, \mathscr{A}_{Q}\right) \text { with compact support. }
$$

Obviously a $Q$-semielliptic quadratic integrand is $k$-semielliptic for every $k \leq Q$. We now show that $Q$-semiellipticity and quasiconvexity coincide. Indeed, consider a linear map $x \mapsto L \cdot x$ and a Lipschitz $k$-valued function $g(x)=\sum_{i=1}^{k} \llbracket f_{i}(x)+L \cdot x \rrbracket$, 
where $f=\sum_{i} \llbracket f_{i} \rrbracket$ is compactly supported in $C_{1}$ and $k \leq Q$. Recall the notation $\eta \circ f=k^{-1} \sum_{i} f_{i}$ and the chain rule formulas in [5, Section 1.3.1]. Then,

$$
\begin{aligned}
E(g) & =E(f)+k\langle A L, L\rangle+2 \int_{C_{1}} \sum_{i}\left\langle A L, D f_{i}\right\rangle \\
& =E(f)+k\langle A L, L\rangle+2 k \int_{C_{1}}\langle A L, D(\eta \circ f)\rangle=E(f)+k\langle A L, L\rangle,
\end{aligned}
$$

where the last equality follows integrating by parts. This equality obviously implies the equivalence of $Q$-semiellipticity and quasiconvexity.

2.1. Sufficiency of quasiconvexity. We prove that, given a sequence $\left(v_{k}\right) \subset$ $W^{1, p}\left(\Omega, \mathscr{A}_{Q}\right)$ weakly converging to $u \in W^{1, p}\left(\Omega, \mathscr{A}_{Q}\right)$ and $f$ as in the statement of Theorem 0.2 , then

$$
F(u) \leq \liminf _{k \rightarrow \infty} F\left(v_{k}\right) .
$$

Up to extracting a subsequence, we may assume that the inferior limit in (2.2) is actually a limit (in what follows, for the sake of convenience, subsequences will never be relabeled). Moreover, using Lemma 1.5, again up to a subsequence, there exists $\left(u_{k}\right)$ such that (i)-(iii) in Lemma 1.5 hold. If we prove

$$
F(u) \leq \lim _{k \rightarrow \infty} F\left(u_{k}\right)
$$

then (2.2) follows, since, by the equi-integrability properties (ii) and (iii),

$$
\begin{aligned}
F\left(u_{k}\right) & =\int_{\left\{v_{k}=u_{k}\right\}} f\left(x, v_{k}, D v_{k}\right)+\int_{\left\{v_{k} \neq u_{k}\right\}} f\left(x, u_{k}, D u_{k}\right) \\
& \leq F\left(v_{k}\right)+C \int_{\left\{v_{k} \neq u_{k}\right\}}\left(1+\left|u_{k}\right|^{q}+\left|D u_{k}\right|^{p}\right)=F\left(v_{k}\right)+o(1) .
\end{aligned}
$$

For the sequel, we will fix a function $\varphi:[0,+\infty) \rightarrow[0,+\infty]$ superlinear at infinity such that

$$
\sup _{k} \int_{\Omega}\left(\varphi\left(\left|u_{k}\right|^{q}\right)+\varphi\left(\left|D u_{k}\right|^{p}\right)\right) d x<+\infty .
$$

In order to prove (2.3), it suffices to show that there exists a subset of full measure $\tilde{\Omega} \subseteq \Omega$ such that for $x_{0} \in \tilde{\Omega}$ we have

$$
f\left(x_{0}, u\left(x_{0}\right), D u\left(x_{0}\right)\right) \leq \frac{d \mu}{d \mathscr{L}^{m}}\left(x_{0}\right),
$$

where $\mu$ is the weak ${ }^{*}$ limit in the sense of measure of any converging subsequence of $\left(f\left(x, u_{k}, D u_{k}\right) \mathscr{L}^{m}\llcorner\Omega)\right.$. We choose $\tilde{\Omega}$ to be the set of points $x_{0}$ which satisfy (1.3) in Lemma 1.4 and, for a fixed subsequence with $\left(\varphi\left(\left|u_{k}\right|^{q}\right)+\varphi\left(\left|D u_{k}\right|^{p}\right)\right) \mathscr{L}^{m}\left\llcorner\Omega \rightarrow^{*} \nu\right.$, satisfy

$$
\frac{d \nu}{d \mathscr{L}^{m}}\left(x_{0}\right)<+\infty
$$

Note that such $\tilde{\Omega}$ has full measure by the standard Lebesgue differentiation theory of measure and Lemma 1.4.

We prove (2.5) by a blow-up argument following Fonseca and Müller [6]. Since in the space $\mathscr{A}_{Q}$ translations make sense only for $Q$ multiplicity points, blow-ups of $Q$-valued functions are not well-defined in general. Hence, to carry on this approach, 
we need first to decompose the approximating functions $u_{k}$ according to the structure of the first order-approximation $T_{x_{0}} u$ of the limit, in such a way to reduce to the case of full multiplicity tangent planes.

Claim 1. Let $x_{0} \in \tilde{\Omega}$ and $u\left(x_{0}\right)=\sum_{j=1}^{J} q_{j} \llbracket a_{j} \rrbracket$, with $a_{i} \neq a_{j}$ for $i \neq j$. Then, there exist $\rho_{k} \downarrow 0$ and $\left(w_{k}\right) \subseteq W^{1, \infty}\left(C_{\rho_{k}}\left(x_{0}\right), \mathscr{A}_{Q}\right)$ such that:

(a) $w_{k}=\sum_{j=1}^{J} \llbracket w_{k}^{j} \rrbracket$ with $w_{k}^{j} \in W^{1, \infty}\left(C_{\rho_{k}}\left(x_{0}\right), \mathscr{A}_{q_{j}}\right),\left\|\mathscr{G}\left(w_{k}, u\left(x_{0}\right)\right)\right\|_{L^{\infty}\left(C_{\rho_{k}}\left(x_{0}\right)\right)}=$ $o(1)$ and $\mathscr{G}\left(w_{k}(x), u\left(x_{0}\right)\right)^{2}=\sum_{j=1}^{J} \mathscr{G}\left(w_{k}^{j}(x), q_{j} \llbracket a_{j} \rrbracket\right)^{2}$ for every $x \in C_{\rho_{k}}\left(x_{0}\right)$;

(b) $f_{C_{\rho_{k}}\left(x_{0}\right)} \mathscr{G}^{p}\left(w_{k}, T_{x_{0}} u\right)=o\left(\rho_{k}^{p}\right)$;

(c) $\lim _{k \uparrow+\infty} f_{C_{\rho_{k}}\left(x_{0}\right)} f\left(x_{0}, u\left(x_{0}\right), D w_{k}\right)=\frac{d \mu}{d \mathscr{L}^{m}}\left(x_{0}\right)$.

Proof. We choose radii $\rho_{k}$ which satisfy the following conditions:

$$
\begin{gathered}
\sup _{k} f_{C_{\rho_{k}}\left(x_{0}\right)}\left(\varphi\left(\left|u_{k}\right|^{q}\right)+\varphi\left(\left|D u_{k}\right|^{p}\right)\right)<+\infty, \\
f_{C_{\rho_{k}}\left(x_{0}\right)} f\left(x, u_{k}, D u_{k}\right) \rightarrow \frac{d \mu}{d \mathscr{L}^{m}}\left(x_{0}\right), \\
f_{C_{\rho_{k}}\left(x_{0}\right)} \mathscr{G}^{p}\left(u_{k}, u\right)=o\left(\rho_{k}^{p}\right) \text { and } f_{C_{\rho_{k}}\left(x_{0}\right)} \mathscr{G}^{p}\left(u_{k}, T_{x_{0}} u\right)=o\left(\rho_{k}^{p}\right) .
\end{gathered}
$$

As for (2.7) and (2.8), since

$$
\left(\varphi\left(\left|u_{k}\right|^{q}\right)+\varphi\left(\left|D u_{k}\right|^{p}\right)\right) \mathscr{L}^{m}\left\llcorner\Omega \longrightarrow ^ { * } \nu \quad \text { and } \quad f ( x , u _ { k } , D u _ { k } ) \mathscr { L } ^ { m } \left\llcorner\Omega \longrightarrow^{*} \mu,\right.\right.
$$

we only need to check that $\nu\left(\partial C_{\rho_{k}}\left(x_{0}\right)\right)=\mu\left(\partial C_{\rho_{k}}\left(x_{0}\right)\right)=0$ (see for instance Proposition 2.7 of [3]). Fixed such radii, for every $k$ we can choose a term in the sequence $\left(u_{k}\right)$ in such a way that the first half of $(2.9)$ holds (because of the strong convergence of $\left(u_{k}\right)$ to $\left.u\right)$ : the second half is, hence, consequence of (1.3).

Set $r_{k}=2|D u|\left(x_{0}\right) \rho_{k}$ and consider the retraction maps $\vartheta_{k}: \mathscr{A}_{Q} \rightarrow \bar{B}_{r_{k}}\left(u\left(x_{0}\right)\right) \subset$ $\mathscr{A}_{Q}$ constructed in [5, Lemma 3.7] (note that for $k$ sufficiently large, these maps are well defined). The functions $w_{k}:=\vartheta_{k} \circ u_{k}$ satisfy the conclusions of the claim.

Indeed, since $\vartheta_{k}$ takes values in $\bar{B}_{r_{k}}\left(u\left(x_{0}\right)\right) \subset \mathscr{A}_{Q}$ and $r_{k} \rightarrow 0$, (a) follows straightforwardly. As for (b), the choice of $r_{k}$ implies that $\vartheta_{k} \circ T_{x_{0}} u=T_{x_{0}} u$ on $C_{\rho_{k}}\left(x_{0}\right)$, because

$$
\mathscr{G}\left(T_{x_{0}} u(x), u\left(x_{0}\right)\right) \leq\left|D u\left(x_{0}\right)\right|\left|x-x_{0}\right| \leq\left|D u\left(x_{0}\right)\right| \rho_{k}=\frac{r_{k}}{2} .
$$

Hence, being $\operatorname{Lip}\left(\vartheta_{k}\right) \leq 1$, from $(2.9)$ we conclude

$$
f_{C_{\rho_{k}}\left(x_{0}\right)} \mathscr{G}^{p}\left(w_{k}, T_{x_{0}} u\right)=f_{C_{\rho_{k}}\left(x_{0}\right)} \mathscr{G}^{p}\left(\vartheta_{k} \circ u_{k}, \vartheta_{k} \circ T_{x_{0}} u\right) \leq f_{C_{\rho_{k}}\left(x_{0}\right)} \mathscr{G}^{p}\left(u_{k}, T_{x_{0}} u\right)=o\left(\rho_{k}^{p}\right) .
$$

To prove (c), set $A_{k}=\left\{w_{k} \neq u_{k}\right\}=\left\{\mathscr{G}\left(u_{k}, u\left(x_{0}\right)\right)>r_{k}\right\}$ and note that, by Chebychev's inequality, we have

$$
\begin{gathered}
r_{k}^{p} \mathscr{L}^{m}\left(A_{k}\right) \leq \int_{A_{k}} \mathscr{G}^{p}\left(u_{k}, u\left(x_{0}\right)\right) \leq 2^{p-1} \int_{A_{k}} \mathscr{G}^{p}\left(u_{k}, T_{x_{0}} u\right)+2^{p-1} \int_{A_{k}} \mathscr{G}^{p}\left(T_{x_{0}} u, u\left(x_{0}\right)\right) \\
\stackrel{(2.9),(2.10)}{\leq} o\left(\rho_{k}^{m+p}\right)+\frac{r_{k}^{p}}{2} \mathscr{L}^{m}\left(A_{k}\right),
\end{gathered}
$$


which in turn implies

$$
\mathscr{L}^{m}\left(A_{k}\right)=o\left(\rho_{k}^{m}\right)
$$

Using Lemma 1.6, we prove that

$$
\lim _{k \rightarrow+\infty}\left(f_{C_{\rho_{k}}\left(x_{0}\right)} f\left(x_{0}, u\left(x_{0}\right), D w_{k}\right)-f_{C_{\rho_{k}}\left(x_{0}\right)} f\left(x, w_{k}, D w_{k}\right)\right)=0 .
$$

Indeed, for every $t>0$,

$$
\begin{aligned}
& \left|f_{C_{\rho_{k}}\left(x_{0}\right)} f\left(x_{0}, u\left(x_{0}\right), D w_{k}\right)-f_{C_{\rho_{k}}\left(x_{0}\right)} f\left(x, w_{k}, D w_{k}\right)\right| \\
& \leq \rho_{k}^{-m} \int_{C_{\rho_{k}}\left(x_{0}\right) \cap\left\{\left|D w_{k}\right| \geq t\right\}}\left(f\left(x_{0}, u\left(x_{0}\right), D w_{k}\right)+f\left(x, w_{k}, D w_{k}\right)\right) \\
& \quad+\rho_{k}^{-m} \int_{C_{\rho_{k}}\left(x_{0}\right) \cap\left\{\left|D w_{k}\right|<t\right\}}\left|f\left(x_{0}, u\left(x_{0}\right), D w_{k}\right)-f\left(x, w_{k}, D w_{k}\right)\right| \\
& \leq \sup _{k} \frac{C}{\rho_{k}^{m}} \int_{C_{\rho_{k}}\left(x_{0}\right) \cap\left\{\left|D w_{k}\right| \geq t\right\}}\left(1+\left|w_{k}\right|^{q}+\left|D w_{k}\right|^{p}\right)+\omega_{f, t}\left(\rho_{k}+\| \mathscr{G}\left(w_{k}, u\left(x_{0}\right) \|_{L^{\infty}}\right),\right.
\end{aligned}
$$

where $\omega_{f, t}$ is a modulus of continuity for $f$ restricted to the compact set $\bar{C}_{\rho_{1}}\left(x_{0}\right) \times$ $\bar{B}_{\left|u\left(x_{0}\right)\right|+1} \times \bar{B}_{t} \subset \Omega \times\left(\mathbf{R}^{n}\right)^{Q} \times\left(\mathbf{R}^{m+n}\right)^{Q}$. To fully justify the last inequality we remark that we choose the same order of the gradients in both integrands so that the order for $u\left(x_{0}\right)$ and for $w_{k}$ is the one giving the $L^{\infty}$ distance between them. Then, (2.12) follows by passing to the limit in (2.13) first as $k \rightarrow+\infty$ and then as $t \rightarrow+\infty$ thanks to (1.6) in Lemma 1.6 applied to $1+\left|w_{k}\right|^{q}$ (which is equi-bounded in $L^{\infty}\left(C_{\rho_{k}}\left(x_{0}\right)\right.$ ) and, hence, equi-integrable) and to $\left|D w_{k}\right|^{p}$.

Thus, in order to show item (c), it suffices to prove

$$
\lim _{k \rightarrow+\infty}\left(f_{C_{\rho_{k}}\left(x_{0}\right)} f\left(x, u_{k}, D u_{k}\right)-f_{C_{\rho_{k}}\left(x_{0}\right)} f\left(x, w_{k}, D w_{k}\right)\right)=0 .
$$

By the definition of $A_{k}$, we have

$$
\begin{aligned}
& \left|f_{C_{\rho_{k}}\left(x_{0}\right)} f\left(x, u_{k}, D u_{k}\right)-f_{C_{\rho_{k}}\left(x_{0}\right)} f\left(x, w_{k}, D w_{k}\right)\right| \\
& \leq \rho_{k}^{-m} \int_{A_{k}}\left(f\left(x, u_{k}, D u_{k}\right)+f\left(x, w_{k}, D w_{k}\right)\right) \\
& \leq \frac{C}{\rho_{k}^{m}} \int_{A_{k}}\left(1+\left|w_{k}\right|^{q}+\left|u_{k}\right|^{q}+\left|D w_{k}\right|^{p}+\left|D u_{k}\right|^{p}\right) .
\end{aligned}
$$

Hence, by the equi-integrability of $u_{k}, w_{k}$ and their gradients, and by (2.11), we can conclude from (1.7) of Lemma 1.6

Using Claim 1, we can now "blow-up" the functions $w_{k}$ and conclude the proof of (2.5). More precisely we will show: 
Claim 2. For every $\gamma>0$, there exist $\left(z_{k}\right) \subset W^{1, \infty}\left(C_{1}, \mathscr{A}_{Q}\right)$ such that $\left.z_{k}\right|_{\partial C_{1}}=$ $\left.T_{x_{0}} u\right|_{\partial C_{1}}$ for every $k$ and

$$
\limsup _{k \rightarrow+\infty} \int_{C_{1}} f\left(x_{0}, u\left(x_{0}\right), D z_{k}\right) \leq \frac{d \mu}{d \mathscr{L}^{m}}\left(x_{0}\right)+\gamma .
$$

Assuming the claim and testing the definition of quasiconvexity of $f\left(x_{0}, \cdot, \cdot\right)$ through the $z_{k}$ 's, by (2.15), we get

$$
f\left(x_{0}, u\left(x_{0}\right), D u\left(x_{0}\right)\right) \leq \limsup _{k \rightarrow+\infty} \int_{C_{1}} f\left(x_{0}, u\left(x_{0}\right), D z_{k}\right) \leq \frac{d \mu}{d \mathscr{L}^{m}}\left(x_{0}\right)+\gamma,
$$

which implies (2.5) by letting $\gamma \downarrow 0$ and concludes the proof.

Proof of Claim 2. We consider the functions $w_{k}$ of Claim 1 and, since they have full multiplicity at $x_{0}$, we can blow-up. Let $\zeta_{k}:=\sum_{j=1}^{J} \llbracket \zeta_{k}^{j} \rrbracket$ with the maps $\zeta_{k}^{j} \in W^{1, \infty}\left(C_{1}, \mathscr{A}_{q_{j}}\right)$ defined by $\zeta_{k}^{j}(y):=\tau_{-a_{j}}\left(\rho_{k}^{-1} \tau_{a_{j}}\left(w_{k}^{j}\right)\left(x_{0}+\rho_{k} \cdot\right)\right)(y)$, with $\tau_{-a_{j}}$ defined in (1.1). Clearly, a simple change of variables gives

$$
\zeta_{k}^{j} \rightarrow q_{j} \llbracket a_{j}+L_{j} \rrbracket \quad \text { in } L^{p}\left(C_{1}, \mathscr{A}_{q_{j}}\right)
$$

and, by Claim 1 (c),

$$
\lim _{k \rightarrow+\infty} \int_{C_{1}} f\left(x_{0}, u\left(x_{0}\right), D \zeta_{k}\right)=\frac{d \mu}{d \mathscr{L}^{m}}\left(x_{0}\right) .
$$

Now, we modify the sequence $\left(\zeta_{k}\right)$ into a new sequence $\left(z_{k}\right)$ in order to satisfy the boundary conditions and (2.15). For every $\delta>0$, we find $r \in(1-\delta, 1)$ such that

$$
\liminf _{k \rightarrow+\infty} \int_{\partial C_{r}}\left|D \zeta_{k}\right|^{p} \leq \frac{C}{\delta} \quad \text { and } \quad \lim _{k \rightarrow+\infty} \int_{\partial C_{r}} \mathscr{G}^{p}\left(\zeta_{k}, T_{x_{0}} u\right)=0
$$

Indeed, by using Fatou's lemma, we have

$$
\begin{gathered}
\int_{1-\delta}^{1} \liminf _{k \rightarrow+\infty} \int_{\partial C_{s}}\left|D \zeta_{k}\right|^{p} d s \leq \liminf _{k \rightarrow+\infty} \int_{C_{1} \backslash C_{1-\delta}}\left|D \zeta_{k}\right|^{p} \leq C \\
\int_{1-\delta}^{1} \lim _{k \rightarrow+\infty} \int_{\partial C_{s}} \mathscr{G}^{p}\left(\zeta_{k}, T_{x_{0}} u\right) d s \leq \liminf _{k \rightarrow+\infty} \int_{C_{1} \backslash C_{1-\delta}} \mathscr{G}^{p}\left(\zeta_{k}, T_{x_{0}} u\right) \stackrel{(2.16)}{=} 0
\end{gathered}
$$

which together with the mean value theorem gives (2.18). Then we fix $\varepsilon>0$ such that $r(1+\varepsilon)<1$ and we apply the interpolation result [5, Lemma 2.15] to infer the existence of a function $z_{k} \in W^{1, \infty}\left(C_{1}, \mathscr{A}_{Q}\right)$ such that $\left.z_{k}\right|_{C_{r}}=\left.\zeta_{k}\right|_{C_{r}},\left.z_{k}\right|_{C_{1} \backslash C_{r(1+\varepsilon)}}=$ $\left.T_{x_{0}} u\right|_{C_{1} \backslash C_{r(1+\varepsilon)}}$ and

$$
\int_{C_{r(1+\varepsilon) \backslash C_{r}}}\left|D z_{k}\right|^{p} \leq C \varepsilon r\left(\int_{\partial C_{r}}\left|D \zeta_{k}\right|^{p}+\int_{\partial C_{r}}\left|D T_{x_{0}} u\right|^{p}\right)+\frac{C}{\varepsilon r} \int_{\partial C_{r}} \mathscr{G}^{p}\left(\zeta_{k}, T_{x_{0}} u\right)
$$

$$
\leq C \varepsilon\left(1+\delta^{-1}\right)+\frac{C}{\varepsilon} \int_{\partial C_{r}} \mathscr{G}^{p}\left(\zeta_{k}, T_{x_{0}} u\right) .
$$


Therefore, by (2.19), we infer

$$
\begin{aligned}
& \int_{C_{1}} f\left(x_{0}, u\left(x_{0}\right), D z_{k}\right)=\int_{C_{r}} f\left(x_{0}, u\left(x_{0}\right), D \zeta_{k}\right) \\
& \quad+\int_{C_{r(1+\varepsilon)} \backslash C_{r}} f\left(x_{0}, u\left(x_{0}\right), D z_{k}\right)+\int_{C_{1} \backslash C_{r(1+\varepsilon)}} f\left(x_{0}, u\left(x_{0}\right), D u\left(x_{0}\right)\right) \\
& \leq \int_{C_{1}} f\left(x_{0}, u\left(x_{0}\right), D \zeta_{k}\right)+C \varepsilon\left(1+\delta^{-1}\right)+\frac{C}{\varepsilon} \int_{\partial C_{r}} \mathscr{G}^{p}\left(\zeta_{k}, T_{x_{0}} u\right)+C \delta .
\end{aligned}
$$

Choosing $\delta>0$ and $\varepsilon>0$ such that $C \varepsilon\left(1+\delta^{-1}\right)+C \delta \leq \gamma$, and taking the superior limit as $k$ goes to $+\infty$ in the latter inequality, we get (2.15) thanks to (2.17) and (2.18).

2.2. Necessity of quasiconvexity. We now prove that, if $F$ is weak ${ }^{*}-W^{1, \infty}$ lower semicontinuous, then $f\left(x_{0}, \cdot, \cdot\right)$ is $Q$-quasiconvex for every $x_{0} \in \Omega$. Without loss of generality, assume $x_{0}=0$ and fix an affine $Q$-function $u$ and functions $w^{j}$ as in Definition 0.1. Set $z^{j}(y):=\sum_{i=1}^{q_{j}} \llbracket\left(w^{j}(y)\right)_{i}-a_{j}-L_{j} \cdot y \rrbracket$, so that $\left.z^{j}\right|_{\partial C_{1}}=q_{j} \llbracket 0 \rrbracket$, and extend it by $C_{1}$-periodicity.

We consider $v_{k}^{j}(y)=\sum_{i=1}^{q_{j}} \llbracket k^{-1}\left(z^{j}(k y)\right)_{i}+a_{j}+L_{j} \cdot y \rrbracket$ and, for every $r>0$ such that $C_{r} \subseteq \Omega$, we define $u_{k, r}(x)=\sum_{j=1}^{J} \tau_{(r-1) a_{j}}\left(r v_{k}^{j}\left(r^{-1} x\right)\right)$. Note that:

(a) for every $r, u_{k, r} \rightarrow u$ in $L^{\infty}\left(C_{r}, \mathscr{A}_{Q}\right)$ as $k \rightarrow+\infty$;

(b) $\left.u_{k, r}\right|_{\partial C_{r}}=\left.u\right|_{\partial C_{r}}$ for every $k$ and $r$;

(c) for every $k, u_{k, r}(0)=\sum_{j=1}^{J} \tau_{-a_{j}}\left(r / k z^{j}(0)\right) \rightarrow u(0)$ as $r \rightarrow 0$;

(d) for every $r, \sup _{k}|| D u_{k, r} \mid \|_{L^{\infty}\left(C_{r}\right)}<+\infty$, since

$$
\left|D u_{k, r}\right|^{2}(x)=\sum_{j=1}^{J}\left|D v_{k}^{j}\right|^{2}\left(r^{-1} x\right)=\sum_{j=1}^{J} \sum_{i=1}^{q_{j}}\left|D z_{i}^{j}\left(k r^{-1} x\right)+L_{j}\right|^{2} .
$$

From (a) and (d) it follows that, for every $r, u_{k, r} \rightarrow^{*} u$ in $W^{1, \infty}\left(C_{r}, \mathscr{A}_{Q}\right)$ as $k \rightarrow+\infty$. Then, by (b), setting $u_{k, r}=u$ on $\Omega \backslash C_{r}$, the lower semicontinuity of $F$ implies that

$$
F\left(u, C_{r}\right):=\int_{C_{r}} f(x, u, D u) \leq \liminf _{k \rightarrow+\infty} F\left(u_{k, r}, C_{r}\right) .
$$

By the definition of $u_{k, r}$, changing the variables in (2.20), we get

$$
\begin{aligned}
& \int_{C_{1}} f(r y, \underbrace{a_{1}+r L_{1} \cdot y}_{q_{1}}, \ldots, \underbrace{a_{J}+r L_{J} \cdot y}_{q_{J}}, L_{1}, \ldots, L_{J}) d y \\
& \leq \liminf _{k \rightarrow \infty} \int_{C_{1}} f\left(r y, \tau_{(r-1) a_{1}}\left(r v_{k}^{1}(y)\right), \ldots, \tau_{(r-1) a_{J}}\left(r v_{k}^{J}(y)\right), D v_{k}^{1}(y), \ldots, D v_{k}^{J}(y)\right) d y .
\end{aligned}
$$

Noting that $\tau_{(r-1) a_{j}}\left(r v_{k}^{j}(y)\right) \rightarrow q_{j} \llbracket a_{j} \rrbracket$ in $L^{\infty}\left(C_{1}, \mathscr{A}_{q_{j}}\right)$ as $r$ tends to 0 and $D v_{k}^{j}(y)=$ $\tau_{-L_{j}}\left(D z^{j}(k y)\right),(2.21)$ leads to

$$
\begin{aligned}
& f(0, \underbrace{a_{1}, \ldots, a_{1}}_{q_{1}}, \ldots, \underbrace{a_{J}, \ldots, a_{J}}_{q_{J}}, L_{1}, \ldots, L_{J}) \\
& \leq \liminf _{k \rightarrow \infty} \int_{C_{1}} f(0, \underbrace{a_{1}, \ldots, a_{1}}_{q_{1}}, \ldots, \underbrace{a_{J}, \ldots, a_{J}}_{q_{J}}, \tau_{-L_{1}}\left(D z^{1}(k y)\right), \ldots, \tau_{-L_{J}}\left(D z^{J}(k y)\right)) d y .
\end{aligned}
$$


Using the periodicity of $z^{j}$, the integral on the right hand side of (2.22) equals

$$
\int_{C_{1}} f(0, \underbrace{a_{1}, \ldots, a_{1}}_{q_{1}}, \ldots, \underbrace{a_{J}, \ldots, a_{J}}_{q_{J}}, \tau_{-L_{1}}\left(D z^{1}(y)\right), \ldots, \tau_{-L_{J}}\left(D z^{J}(y)\right)) d y .
$$

Since $\tau_{-L_{j}}\left(D z^{j}\right)=D w^{j}$, we conclude $(0.2)$.

\section{Polyconvexity}

In this section we prove Theorem 0.5 and show the semicontinuity of Almgren's Dirichlet energy and Mattila's quadratic energies. Recall the notation for multiindices and minors $M_{\alpha, \beta}$ introduced in Section 1.

Definition 3.1. A map $P: \mathbf{R}^{n \times m} \rightarrow \mathbf{R}$ is polyaffine if there are constants $c_{0}, c_{\alpha \beta}^{l}$, for $l \in\{1, \ldots, N\}$ and $\alpha, \beta$ multi-indices, such that

$$
P(A)=c_{0}+\sum_{l=1}^{N} \sum_{|\alpha|=|\beta|=l} c_{\alpha \beta}^{l} M_{\alpha \beta}(A)=c_{0}+\langle\zeta, M(A)\rangle,
$$

where $\zeta \in \mathbf{R}^{\tau(m, n)}$ is the vector whose entries are the $c_{\alpha \beta}^{l}$ 's and $M(A)$ is the vector of all minors.

It is possible to represent polyconvex functions as supremum of a family of polyaffine functions retaining some symmetries from the invariance of $f$ under the action of permutations.

Proposition 3.2. Let $f$ be a $Q$-integrand, then the following are equivalent:

(i) $f$ is a polyconvex $Q$-integrand,

(ii) for every choice of vectors $a_{1}, \ldots, a_{Q} \in \mathbf{R}^{n}$ and matrices $A_{1}, \ldots A_{Q} \in \mathbf{R}^{n \times m}$, with $A_{i}=A_{j}$ if $a_{i}=a_{j}$, there exist polyaffine functions $P_{j}: \mathbf{R}^{n \times m} \rightarrow \mathbf{R}$, with $P_{i}=P_{j}$ if $a_{i}=a_{j}$, such that

$$
f\left(a_{1}, \ldots, a_{Q}, A_{1}, \ldots, A_{Q}\right)=\sum_{j=1}^{Q} P_{j}\left(A_{j}\right)
$$

and

$$
f\left(a_{1}, \ldots, a_{Q}, L_{1}, \ldots, L_{Q}\right) \geq \sum_{j=1}^{Q} P_{j}\left(L_{j}\right) \quad \text { for every } L_{1}, \ldots, L_{Q} \in \mathbf{R}^{n \times m} .
$$

Proof. (i) $\Rightarrow$ (ii). Let $g$ be a function representing $f$ according to Definition 0.4. Convexity of the subdifferential of $g\left(a_{1}, \ldots, a_{Q}, \cdot\right)$, condition $(0.3)$ and the invariance of $f$ under the action of permutations yield that there exists $\zeta \in \partial g\left(a_{1}, \ldots, a_{Q}\right.$, $\left.M\left(A_{1}\right), \ldots, M\left(A_{Q}\right)\right)$, with $\zeta_{i}=\zeta_{j}$ if $a_{i}=a_{j}$, such that for every $X \in\left(\mathbf{R}^{\tau(m, n)}\right)^{Q}$ we have

$$
\begin{aligned}
& g\left(a_{1}, \ldots, a_{Q}, X_{1}, \ldots, X_{Q}\right) \\
& \geq g\left(a_{1}, \ldots, a_{Q}, M\left(A_{1}\right), \ldots, M\left(A_{Q}\right)\right)+\sum_{j=1}^{Q}\left\langle\zeta_{j}, X_{j}-M\left(A_{j}\right)\right\rangle .
\end{aligned}
$$

Hence, the maps $P_{j}: \mathbf{R}^{n \times m} \rightarrow \mathbf{R}$ given by

$$
P_{j}(L):=Q^{-1} g\left(a_{1}, \ldots, a_{Q}, M\left(A_{1}\right), \ldots, M\left(A_{Q}\right)\right)+\left\langle\zeta_{j}, M(L)-M\left(A_{j}\right)\right\rangle
$$


are polyaffine and such that (3.2) and (3.3) follow. that

(ii) $\Rightarrow\left(\right.$ i). By (3.2) and (3.3), there exists $\zeta_{j}$, satisfying $\zeta_{i}=\zeta_{j}$ if $a_{i}=a_{j}$, such

$$
\begin{aligned}
& f\left(a_{1}, \ldots, a_{Q}, L_{1}, \ldots, L_{Q}\right) \\
& \geq f\left(a_{1}, \ldots, a_{Q}, A_{1}, \ldots, A_{Q}\right)+\sum_{j=1}^{Q}\left\langle\zeta_{j}, M\left(L_{j}\right)-M\left(A_{j}\right)\right\rangle .
\end{aligned}
$$

Then setting,

$$
g\left(a_{1}, \ldots, a_{Q}, X_{1}, \ldots, X_{Q}\right):=\sup \left\{f\left(a_{1}, \ldots, a_{Q}, A_{1}, \ldots, A_{Q}\right)+\sum_{j=1}^{Q}\left\langle\zeta_{j}, X_{j}-M\left(A_{j}\right)\right\rangle\right\}
$$

where the supremum is taken over all $A_{1}, \ldots, A_{Q} \in \mathbf{R}^{n \times m}$ with $A_{i}=A_{j}$ if $a_{i}=a_{j}$, it follows clearly that $g\left(a_{1}, \ldots, a_{Q}, \cdot\right)$ is a convex function and $(0.3)$ holds thanks to (3.6). In turn, these remarks and the equality $\operatorname{co}\left(\left(M\left(\mathbf{R}^{n \times m}\right)\right)^{Q}\right)=\left(\mathbf{R}^{\tau(m, n)}\right)^{Q}$ imply that $g\left(a_{1}, \ldots, a_{Q}, \cdot\right)$ is everywhere finite.

We are now ready for the proof of Theorem 0.5.

Proof of Theorem 0.5. Assume that $f$ is a polyconvex $Q$-integrand and consider $a_{j}, L_{j}$ and $w^{j}$ as in Definition 0.1. Corresponding to this choice, by Proposition 3.2, there exist polyaffine functions $P_{j}$ satisfying (3.2) and (3.3), which read as

$$
f(\underbrace{a_{1}, \ldots, a_{1}}_{q_{1}}, \ldots, \underbrace{a_{J}, \ldots, a_{J}}_{q_{J}}, \underbrace{L_{1}, \ldots, L_{1}}_{q_{1}}, \ldots \underbrace{L_{J}, \ldots, L_{J}}_{q_{J}})=\sum_{j=1}^{J} q_{j} P_{j}\left(L_{j}\right)
$$

and, for every $B_{1}, \ldots, B_{Q} \in \mathbf{R}^{m \times n}$,

$$
f(\underbrace{a_{1}, \ldots, a_{1}}_{q_{1}}, \ldots, \underbrace{a_{J}, \ldots, a_{J}}_{q_{J}}, B_{1}, \ldots, B_{Q}) \geq \sum_{j=1}^{J}\left\{\sum_{i=\sum_{l<j} q_{l}+1}^{\sum_{l \leq j} q_{l}} P_{j}\left(B_{i}\right)\right\} .
$$

To prove the theorem it is enough to show that

$$
\sum_{j=1}^{J} q_{j} P_{j}\left(L_{j}\right)=\int_{C_{1}} \sum_{j=1}^{J} \sum_{i=1}^{q_{j}} P_{j}\left(D w_{i}^{j}\right) .
$$

Indeed, then the quasiconvexity of $f$ follows easily from

$$
\begin{aligned}
& f(\underbrace{a_{1}, \ldots, a_{1}}_{q_{1}}, \ldots, \underbrace{a_{J}, \ldots, a_{J}}_{q_{J}}, \underbrace{L_{1}, \ldots, L_{1}}_{q_{1}}, \ldots \underbrace{L_{J}, \ldots, L_{J}}_{q_{J}}) \stackrel{(3.2)}{=} \sum_{j=1}^{J} q_{j} P_{j}\left(L_{j}\right) \\
& \stackrel{(3.9)}{=} \int_{C_{1}} \sum_{j=1}^{J} \sum_{i=1}^{q_{j}} P_{j}\left(D w_{i}^{j}\right) \stackrel{(3.3)}{\leq} \int_{C_{1}} f(\underbrace{a_{1}, \ldots, a_{1}}_{q_{1}}, \ldots, \underbrace{a_{J}, \ldots, a_{J}}_{q_{J}}, D w^{1}, \ldots, D w^{J}) .
\end{aligned}
$$

To prove (3.9), consider the current $T_{w^{j}, C_{1}}$ associated to the graph of the $q_{j^{-}}$valued map $w^{j}$ and note that, by definition (1.10), for the exact, constant coefficient $m$-form 
$d \omega^{j}=c_{0}^{j} d x+\sum_{l=1}^{N} \sum_{|\alpha|=|\beta|=l} \sigma_{\alpha} c_{\alpha \beta}^{j, l} d x_{\bar{\alpha}} \wedge d y_{\beta}$, it holds

$$
\int_{C_{1}} \sum_{i=1}^{q_{j}} P_{j}\left(D w_{i}^{j}\right)=\left\langle T_{w^{j}, C_{1}}, d \omega^{j}\right\rangle,
$$

where $P_{j}(A)=c_{0}^{j}+\sum_{l=1}^{N} \sum_{|\alpha|=|\beta|=l} c_{\alpha \beta}^{j, l} M_{\alpha \beta}(A)$.

Since $\left.u\right|_{\partial C_{1}}=\left.w\right|_{\partial C_{1}}$, from Theorem 1.7 it follows that $\partial T_{w, C_{1}}=\partial T_{u, C_{1}}$. Then, (3.9) is an easy consequence of (3.10): for $u^{j}(x)=q_{j} \llbracket a_{j}+L_{j} \cdot x \rrbracket$, one has, indeed,

$$
\begin{aligned}
\sum_{j=1}^{J} q_{j} P_{j}\left(L_{j}\right) & =\int_{C_{1}} \sum_{j=1}^{J} \sum_{i=1}^{q_{j}} P_{j}\left(D u_{i}^{j}\right)=\sum_{j=1}^{J}\left\langle T_{u^{j}, C_{1}}, d \omega^{j}\right\rangle=\sum_{j=1}^{J}\left\langle\partial T_{u^{j}, C_{1}}, \omega^{j}\right\rangle \\
& =\sum_{j=1}^{J}\left\langle\partial T_{w^{j}, C_{1}}, \omega^{j}\right\rangle=\sum_{j=1}^{J}\left\langle T_{w^{j}, C_{1}}, d \omega^{j}\right\rangle=\int_{C_{1}} \sum_{j=1}^{J} \sum_{i=1}^{q_{j}} P_{j}\left(D w_{i}^{j}\right) .
\end{aligned}
$$

This finishes the proof.

Explicit examples of polyconvex functions are collected below (the elementary proof is left to the reader).

Proposition 3.3. The following classes of functions are polyconvex $Q$-integrands:

(a) $f\left(a_{1}, \ldots, a_{Q}, L_{1}, \ldots, L_{Q}\right):=g(\mathscr{G}(L, Q \llbracket 0 \rrbracket))$ with $g: \mathbf{R} \rightarrow \mathbf{R}$ convex and increasing;

(b) $f\left(a_{1}, \ldots, a_{Q}, L_{1}, \ldots, L_{Q}\right):=\sum_{i, j=1}^{Q} g\left(L_{i}-L_{j}\right)$ with $g: \mathbf{R}^{n \times m} \rightarrow \mathbf{R}$ convex;

(c) $f\left(a_{1}, \ldots, a_{Q}, L_{1}, \ldots, L_{Q}\right):=\sum_{i=1}^{Q} g\left(a_{i}, L_{i}\right)$ with $g: \mathbf{R}^{m} \times \mathbf{R}^{n \times m} \rightarrow \mathbf{R}$ measurable and polyconvex.

Remark 3.4. Consider as in Remark 2.1 a linear symmetric map $\mathbf{R}^{n \times m} \ni M \mapsto$ $A M \in \mathbf{R}^{n \times m}$. As it is well-known, for classical single valued functions the functional

$$
\int\langle A D f, D f\rangle
$$

is quasiconvex if and only if it is rank-1 convex. If $\min \{m, n\} \leq 2$, quasiconvexity is equivalent to polyconvexity as well (see [14]). Hence, in this case, by Theorem 0.5, every 1-semielliptic integrand is quasiconvex and therefore $Q$-semielliptic.

We stress that for $\min \{m, n\} \geq 3$ there exist 1-semielliptic integrands which are not polyconvex (see always [14]).

\section{Appendix A. Equi-integrability}

Let us first recall some definitions and introduce some notation. As usual, in the following $\Omega \subset \mathbf{R}^{m}$ denotes a Lipschitz set with finite measure.

Definition A.1. A sequence $\left(g_{k}\right)$ in $L^{1}(\Omega)$ is equi-integrable if one of the following equivalent conditions holds:

(a) for every $\varepsilon>0$ there exists $\delta>0$ such that, for every $\mathscr{L}^{m}$-measurable set $E \subseteq \Omega$ with $\mathscr{L}^{m}(E) \leq \delta$, we have $\sup _{k} \int_{E}\left|g_{k}\right| \leq \varepsilon$

(b) the distribution functions $\varphi_{k}(t):=\int_{\left\{\left|g_{k}\right| \geq t\right\}}\left|g_{k}\right|$ satisfy $\lim _{t \rightarrow+\infty} \sup _{k} \varphi_{k}(t)=0$; 
(c) (De la Vallée Poissin's criterion) if there exists a Borel function $\varphi:[0,+\infty) \rightarrow$ $[0,+\infty]$ such that

$$
\lim _{t \rightarrow+\infty} \frac{\varphi(t)}{t}=+\infty \quad \text { and } \sup _{k} \int_{\Omega} \varphi\left(\left|g_{k}\right|\right) d x<+\infty .
$$

Note that, since $\Omega$ has finite measure, an equi-integrable sequence is also equibounded. We prove now Chacon's biting lemma.

Lemma A.2. Let $\left(g_{k}\right)$ be a bounded sequence in $L^{1}(\Omega)$. Then, there exist a subsequence $\left(k_{j}\right)$ and a sequence $\left(t_{j}\right) \subset[0,+\infty)$ with $t_{j} \rightarrow+\infty$ such that $\left(g_{k_{j}} \vee\right.$ $\left.\left(-t_{j}\right) \wedge t_{j}\right)$ is equi-integrable.

Proof. Without loss of generality, assume $g_{k} \geq 0$ and consider for every $j \in \mathbf{N}$ the functions $h_{k}^{j}:=g_{k} \wedge j$. Since, for every $j,\left(h_{k}^{j}\right)_{k}$ is equi-bounded in $L^{\infty}$, up to passing to a subsequence (not relabeled), there exists the $L^{\infty}$ weak ${ }^{*}$ limit $f_{j}$ of $h_{k}^{j}$ for every $j$. Clearly the limits $f_{j}$ have the following properties:

(a) $f_{j} \leq f_{j+1}$ for every $j$ (since $h_{k}^{j} \leq h_{k}^{j+1}$ for every $k$ );

(b) $\left\|f_{j}\right\|_{L^{1}}=\lim _{k}\left\|h_{k}^{j}\right\|_{L^{1}}$;

(c) $\sup _{j}\left\|f_{j}\right\|_{L^{1}}=\sup _{j} \lim _{k}\left\|h_{k}^{j}\right\|_{L^{1}} \leq \sup _{k}\left\|g_{k}\right\|_{L^{1}}<+\infty$.

By the Lebesgue monotone convergence theorem, (a) and (c), it follows that $\left(f_{j}\right)$ converges in $L^{1}$ to a function $f$. Moreover, from (b), for every $j$ we can find a $k_{j}$ such that

$$
\left|\int h_{k_{j}}^{j}-\int f_{j}\right| \leq j^{-1}
$$

We claim that $h_{k_{j}}^{j}=g_{k_{j}} \wedge j$ fulfills the conclusion of the lemma (with $t_{j}=j$ ). To see this, it is enough to show that $h_{k_{j}}^{j}$ weakly converges to $f$ in $L^{1}$, from which the equi-integrability follows. Let $a \in L^{\infty}$ be a test function. Since $h_{k_{j}}^{l} \leq h_{k_{j}}^{j}$ for $l \leq j$, we have that

$$
\int\left(\|a\|_{L^{\infty}}-a\right) h_{k_{j}}^{l} \leq \int\left(\|a\|_{L^{\infty}}-a\right) h_{k_{j}}^{j}
$$

Taking the limit as $j$ goes to infinity in (A.3), we obtain (by $h_{k_{j}}^{l} \stackrel{w^{*}-L^{\infty}}{\rightarrow} f_{l}$ and (A.2))

$$
\int\left(\|a\|_{L^{\infty}}-a\right) f_{l} \leq\|a\|_{L^{\infty}} \int f-\limsup _{j} \int a h_{k_{j}}^{j}
$$

From which, passing to the limit in $l$, we conclude since $f_{l} \stackrel{L^{1}}{\rightarrow} f$

$$
\underset{j}{\limsup } \int a h_{k_{j}}^{j} \leq \int a f
$$

Using $-a$ in place of $a$, one obtains as well the inequality

$$
\int a f \leq \liminf _{j} \int a h_{k_{j}}^{j} .
$$

(A.4) and (A.5) together concludes the proof of the weak convergence of $h_{k_{j}}^{j}$ to $f$ in $L^{1}$.

Next we show that concentration effects for critical Sobolev embedding do not show up if equi-integrability of functions and gradients is assumed. 
Lemma A.3. Let $p \in[1, m)$ and $\left(g_{k}\right) \subset W^{1, p}(\Omega)$ be such that $\left(\left|g_{k}\right|^{p}\right)$ and $\left(\left|\nabla g_{k}\right|^{p}\right)$ are both equi-integrable, then $\left(\left|g_{k}\right|^{p^{*}}\right)$ is equi-integrable as well.

Proof. Since $\left(g_{k}\right)$ is bounded in $W^{1, p}(\Omega)$, Chebychev's inequality implies

$$
\sup _{j} j^{p} \mathscr{L}^{m}\left(\left\{\left|g_{k}\right|>j\right\}\right) \leq C<+\infty .
$$

For every fixed $j \in \mathbf{N}$, consider the sequence $g_{k}^{j}:=g_{k}-\left(g_{k} \vee(-j) \wedge j\right)$. Then, $\left(g_{k}^{j}\right) \subset W^{1, p}(\Omega)$ and $\nabla g_{k}^{j}=\nabla g_{k}$ in $\left\{\left|g_{k}\right|>j\right\}$ and $\nabla g_{k}^{j}=0$ otherwise. The Sobolev embedding yields

$$
\left\|g_{k}^{j}\right\|_{L^{p^{*}}(\Omega)}^{p} \leq c\left\|g_{k}^{j}\right\|_{W^{1, p}(\Omega)}^{p} \leq c \int_{\left\{\left|g_{k}\right|>j\right\}}\left(\left|g_{k}\right|^{p}+\left|\nabla g_{k}\right|^{p}\right) d x .
$$

Therefore, the equi-integrability assumptions and (A.6) imply that for every $\varepsilon>0$ there exists $j_{\varepsilon} \in \mathbf{N}$ such that for every $j \geq j_{\varepsilon}$

$$
\sup _{k}\left\|g_{k}^{j}\right\|_{L^{p^{*}}(\Omega)} \leq \varepsilon / 2 .
$$

Let $\delta>0$ and consider a generic $\mathscr{L}^{m}$-measurable sets $E \subseteq \Omega$ with $\mathscr{L}^{m}(E) \leq \delta$. Then, since we have

$$
\left\|g_{k}\right\|_{L^{p^{*}(E)}} \leq\left\|g_{k}-g_{k}^{j_{\varepsilon}}\right\|_{L^{p^{*}}(E)}+\left\|g_{k}^{j_{\varepsilon}}\right\|_{L^{p^{*}(E)}} \leq j_{\varepsilon}\left(\mathscr{L}^{m}(E)\right)^{1 / p^{*}}+\left\|g_{k}^{j_{\varepsilon}}\right\|_{L^{p^{*}}(\Omega)},
$$

by (A.8), to conclude it suffices to choose $\delta$ such that $j_{\varepsilon} \delta^{1 / p^{*}} \leq \varepsilon / 2$.

\section{References}

[1] Almgren, F. J., JR.: Almgren's big regularity paper. - World Sci. Monogr. Ser. Math. 1, World Sci. Publishing, River Edge, NJ, 2000.

[2] Ball, J. M.: Convexity conditions and existence theorems in nonlinear elasticity. - Arch. Rational Mech. Anal. 63:4, 1976/77, 337-403.

[3] De Lellis, C.: Rectifiable sets, densities and tangent measures. - Zurich Lect. in Adv. Math., EMS, Zürich, 2008.

[4] De Lellis, C., and E. N. Spadaro: Higher integrability and approximation of minimal currents. - Preprint, 2009.

[5] De Lellis, C., and E. N. Spadaro: $Q$-valued functions revisited. - Mem. Amer. Math. Soc. (to appear).

[6] Fonseca, I., and S. Müller: Quasi-convex integrands and lower semicontinuity in $L^{1}$. SIAM J. Math. Anal. 23:5, 1992, 1081-1098.

[7] Fonseca, I., S. Müller, and P. Pedregal: Analysis of concentration and oscillation effects generated by gradients. - SIAM J. Math. Anal. 29:3, 1998, 736-756 (electronic).

[8] Giaquinta, M., G. ModicA, and J. SoučEk: Cartesian currents in the calculus of variations. I. Cartesian currents. - Ergeb. Math. Grenzgeb. (3) 37, Springer-Verlag, Berlin, 1998.

[9] Giaquinta, M., G. Modica, and J. Souček: Cartesian currents in the calculus of variations. II. Variational integrals. - Ergeb. Math. Grenzgeb. (3) 38, Springer-Verlag, Berlin, 1998.

[10] Mattila, P.: Lower semicontinuity, existence and regularity theorems for elliptic variational integrals of multiple valued functions. - Trans. Amer. Math. Soc. 280:2, 1983, 589-610.

[11] Morrey, C.B., JR.: Quasi-convexity and the lower semicontinuity of multiple integrals. Pacific J. Math. 2, 1952, 25-53.

[12] Morrey, C. B., JR.: Multiple integrals in the calculus of variations. - Grundlehren Math. Wiss. 130, Springer-Verlag, New York, 1966. 
[13] Stein, E. M.: Harmonic analysis: real-variable methods, orthogonality, and oscillatory integrals. - Princeton Math. Ser. 43, Princeton Univ. Press, Princeton, NJ, 1993.

[14] Terpstra, F. J.: Die Darstellung biquadratischer Formen als Summen von Quadraten mit Anwendung auf die Variationsrechnung. - Math. Ann. 116:1, 1939, 166-180.

Received 9 November 2009 